\title{
Study and Application Analysis of HTML5 Core Technology Based on Mobile Browser
}

\author{
Cuiyu Fu \\ Department of software engineering, Hainan College of Software Technology, Qionghai \\ Hainan ,571400, China
}

Key words: Mobile browser, HTML core technology, Study, Application.

\begin{abstract}
. as internet application develops rapidly, application requirements of internet web becomes increasingly strict. As uses' demand improves, the standard and technology of web browser are changing continuously. HTML5 standard proposed by World Wide Web Consortium (WWWC) offers a new opportunity for internet application and development and promotes users to experience excellent internet web application. This paper deeply studies HTML5 webpage image technology, webpage offline storage technology and webpage video technology etc., expounds application of HTML5 core technology in mobile browser so as to construct web application platform of HTML5 which meets users' demand.
\end{abstract}

\section{Introduction}

WWWC put forward HTML5 standard in 2010. Localization, multi-media and strong interactive technology of the above standard can meet increasing internet application demand. In current stage, mobile internet also develops rapidly. Online game, social media and e-commerce drive the advance of HTML5 technology. To meet the application demand of mutual operation and flexible operation, standardization development of key technology of HTML5 standard is required. More API should be applied to change users' demand for development and application of internet modes and meet users' actual demand.

\section{Basic overview of HTML5}

HTML5 is a language form which reflects and constructs internet content, and it is the standard of next generation of internet. Meanwhile, it is the core technology of internet. HTML technology formed in 1990, and became internet standard in 1997. Besides, it was applied in internet development. HTML5 is the $5^{\text {th }}$ version of HTML. Meanwhile, it is the latest version in the development stage. In a broad sense, HTML5 includes Java Script, CSS, HTML and other combined technologies. Its main function is to reduce rich application services of browser plug-in network, such as the demand for Oracle Java FX and Adobe Flash. Besides, it can offer effective standard set for network application. HTML5 actually integrates many new grammatical features, such as <audio>, $<$ video $>$ and $<$ canvas $>$. Meanwhile, the content of SVG can be integrated to promote to process and add pictures and multimedia in webpage more conveniently. Moreover, HTML5 also remove <big>, $<$ basefont $>$ and other elements and attributes as well as standardizes, rectifies and redefines $<$ cite $>$, $<$ menu $>$ and other elements and attributes. As well, HTML5 analyzes handling of illegal documents so that all client programs and browsers can uniformly handle grammatical mistakes. HTML5 simplifies webpage design, separates the style and layout, reduces script complexity and greatly reduces plug-in dependence.

\section{HTML5 image technology}

Canvas of HTML5 can dynamically form animation, image and graphs and is widely applied in HTML5 games. Hence, it is one of the strongest API. In the process of making webpage images with 
traditional method, it is necessary to complete or directly embed pictures according to the animation, but original Java Script language should be applied in development of canvas pictures. scripting language or Open GL can be called in the page according to Java Script and script language. Besides, 2D image can be made by directly using labels to render 3D effect. Thus, it is very important to apply the above labels in the browser. It is relatively simple to construct $2 \mathrm{D}$ image by canvas. It is just necessary to add corresponding < canvas> label in HTML page. Meanwhile, element ID is set, and the width and height are given to complete canvas content. If the picture is drawn in it, it is necessary to rationally apply Java Script and utilize Element By Id( 'my Canvas')” function to discover canvas element required in initialization. Moreover, API can be applied to draw 2D image. In current stage, API created by 2D image canvas simple shape API, canvas state API, painting brush and failing etc. Meanwhile, drawing method is basically defined. Canvas element analysis has relatively complex API, such as insert image to canvas. During creating 2D image in canvas, similar API is required, such as color gradient API and shadow API. It is very complex to construct 3D image in HTML5. Three kinds of models are basically included. Firstly, Open GL ES and other low-level standard API are used, also called Web GL. Secondly, DOM tree is applied to form DOM tree mapping in X3D scene, also called X3DOM model. Web3D developer alliance has existed for the above models. Thirdly, O3D model is actually the model developed by Google. O3D is the interface of 3D application and mainly uses webpage basic open source program, of Google. If canvas element $r$ is widely applied in mobile browse, mobile users may better experience terminal webgame. Meanwhile, wireless terminal can present 3D animation effect ${ }^{1]}$.

\section{Video technology of HTML5}

Speaking of browser video, we will think of flash technology. HTML5 video technology needs to contend for the market of flash technology. However, PC terminal still uses flash technology in quantity. The biggest problem of application of flash technology in mobile equipment is execution efficiency. But, HTML5 technology owns such advantage. For example, HTML5 video technology is applied in the mobile phone to improve battery cruising power, when HTML5 video technology is applied, < video> label is mainly integrated. Thus, web video can be played without the need of inserting flash plug-in in webpage. Video label mainly includes loop, width, controls, height, src, autoplay and other attributes. Meanwhile, it also contains < source> label. The video to be played can be set through the above labels and attributes. Different browsers need different types of video support software. Phone, Android and iPad also have some differences. Thus, the complexity of < video> improves. Video element supports the video of two kinds of formats such as Ogg and MPEG4. MPEG4 is actually the document with Vorbis audio coding and Thedora video coding. The browser not just needs to interpret < video> label, but also needs to code and decode player video. Thus in this technology, scientific and reasonable code decoder should be selected, and all browsers should be able to execute the above operation. In current stage, when <video> label is applied in HTML5, the following problems should be analyzed. Firstly, browsers supporting H.264 such as Chrome browser and IE9 browser; secondly, browsers supporting VP8 or Ogg Theora such as Chrome browser and Mozilla browser; thirdly, browsers supporting all, such as IE6, 7 and 8 browsers $^{[2]}$.

\section{Offline storage technology and its application in e-learning}

\section{Offline storage technology}

When users cannot connect network, HTML5 technology can be used to better operate webpage offline. HTTP objective can be set to make sure users can visit webpage, set HTTP objective such as configuring HTML file without network. File configuration needs special processing in the development process so as to reach the function of off visit. During offline web program development, the following functions are generally included. Firstly, offline resource cache. In actual development 
process, reasonable manner is needed to reflect the file resource needed in offline application program operation, and to make sure the browser can cache file locally in online state. When users conduct offline visit, the above resource file is loaded automatically, and formally-used resources are provided for users. Cache manifest file is used to indicate the resources cached in HTML5. There are two method to update cache: manual and automatic methods. Secondly, online state detection. System development personnel needs to clearly know the situation of browser and browser to handle the situation of browser in a targeted way. HTML5 includes two methods to detect whether the network is online. Thirdly, store local data. In the process of program offline, it is necessary to store data locally so as to synchronize data in online server. To meet storage requirements of different systems, there are mainly two storage mechanisms Web SQL Database and DOM Storage ${ }^{[3]}$.

Web Storage API proposed by HTML5 mainly includes session Storage and local Storage, and can store simple objects such as application program state to guarantee smooth completion of storage session and local information. But during processing structural data, Web SQL Database API interface should be applied. During network storage in HTML5, there are two types according to HTML5. (1) Session-based storage can only store current session. After session) is closed and website is transferred, stored information will be damaged. (2) local storage. When the webpage is opened again, the webpage which is opened last time can be visited. For example, local username is stored according to the above form. During closing the label page and browsing restarted webpage, the existence of storage is the main difference of the above two types. Local Storage technology is similar to cookies technology. In current stage, such technology has 1000 times of webpage data size stored by cookies, and website virtual seats can be stored on computer. When users visit website and load data, Java Script can be used to visit Local Storage data. But, cookies owns different storage information. During visiting webpage, it is necessary to return cookies data information in web server. Due to the above characteristics, the purpose of offline webpage application can breached. Both local cache and local SQL database can be set. SQL database and local cache can improve the speed of operating web and offer the function of visiting webpage under the condition of network failure ${ }^{[4]}$.

\section{Application of offline storage in e-learning}

With rapid development of science, technology and information technology, e-learning activity becomes increasingly popular. learning management system webpage in academic environment can offer great help for teachers in online test and leanring activity, and gain the approval and favor of people in the industry. But many schools cannot pay corresponding expenses for the above learning management system network in actual operation. Besides, it is very difficult for some students to surf the internet at home. To effectively solve the above problem, webpage storage technology and offline application technology of HTML5 become especially important. Certain system can be utilized to offer offline user interface of mobile browser for students to use the above system. Without network, students can do schoolwork, submit and recover campus moodle task synchronously on mobile phone. After students enter webpage system course, corresponding test tasks can be allocated to them. Students can submit schoolwork offline via mobile phone or do schoolwork online. If mobile phone can be used visit school network through mobile telephone, students can receive synchronous testing according to the test points and do schoolwork at home. Students can complete school test at home through the browser. in addition, students can download in the practice allowed by the system or test. Form the test points, students can gain sole ID, and then gain offline webpage in accordance with URL at desktop client test point. If it is necessary to visit URL, mobile phone can download data and files and complete offline operation application ${ }^{[5]}$.

\section{Conclusion}

In conclusion, HTML5 technology has been widely applied in software development, but it is still in the stage of researching and improving HTML5 technology. The basic objective of HTML5 technology is to research and develop Web network program, offer the next generation of standard, unify page development technology and provide application platform for browser. Currently, 
HTML5 technology is being studied and developed. The study on HTML5 technology mainly focused on development of webpage games, such as Metro Run, webpage cards and 3D building block tower.

\section{References}

[1] Peng Tao, Yang Lian, Study and application of HTML5 core technology based on mobile browser, Guangdong Communication Technology, 2012,32(4):47-50.

[2] Jiang Fucheng, Development and application of HTML5-based webpage map browser, Collected papers of 2014 nationwide academic annual conference about open-type distribution and parallel computing. 2014:364-367.

[3] Zhao Aimei, Study and implementation of mobile learning platform based on HTML5 and .NET, Journal of Henan Institute of Science and Technology (Nature Sciences Edition). 2013,41(4):62-66.

[4] Liu Xiangang, Jiang Yutao, Wu Xinsong wt al., Development status of HTML5 standard and standardization strategy in China, Information Technology \& Standardization, 2012(5):23-27.

[5] Shi Jun, Sang Guoming, Study and development of QtWebkit2.1 inner core based on embedded mobile browser, Computer \& Digital Engineering, 2012,40(3):79-82. 